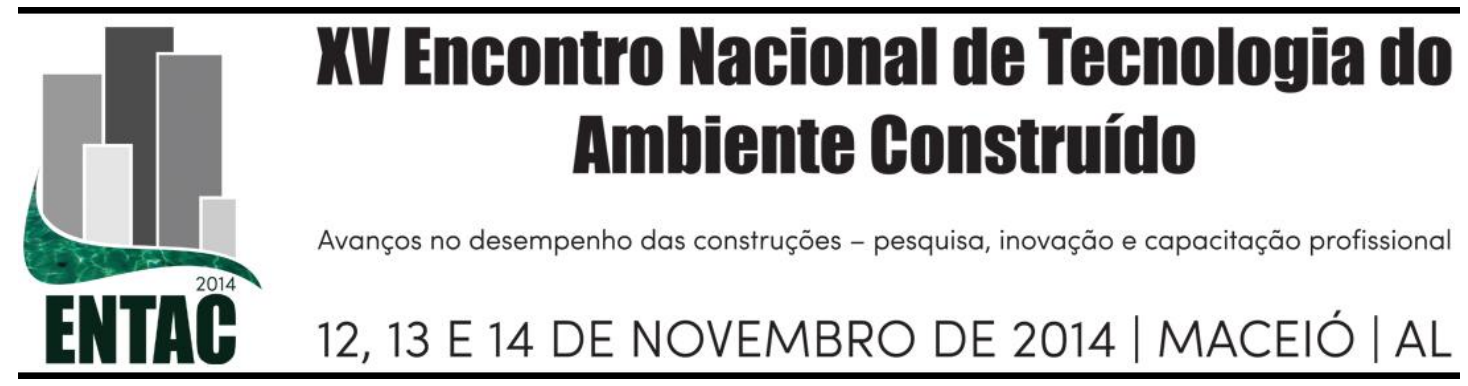

\title{
LINHA DE BALANÇO - SÍNTESE DOS PRINCÍPIOS DE PRODUÇÃO ENXUTA APLICADOS À PROGRAMAÇÃO DE OBRAS?
}

\author{
MOURA, Rafael de Sousa Leal Martins (1); HEINECK, Luiz Fernando \\ Mählmann (2)
}

(1) PEC/UFC, e-mail: rafaelleall@hotmail.com (2)UECE, e-mail: freitas8@terra.com.br

\begin{abstract}
RESUMO
Evidências da utilização da linha de balanço como técnica de programação já existem na realidade brasileira há mais de 30 anos, mas só recentemente ela tem sido associada com aplicações de construção enxuta, notadamente como uma ferramenta para planejamento estratégico de obras. Esse trabalho discute conceitualmente como a linha de balanço pode ser entendida não só como uma forma de graficação das atividades em obra, mas de tal forma que conceitos filosóficos por trás da produção enxuta possam ali estar incorporados ou se tornar evidentes por si só. Algumas características da produção enxuta podem ser aplicadas no desenvolvimento da LOB, tais como a visão de ciclo no tempo e no espaço, simplificação das operações pela externalização ou pela pacotização do trabalho, redução da variabilidade, visão e controle do fluxo de execução da obra, redução do lead time pelo balanceamento das atividades ou pelo efeito aprendizagem e integração de curto, médio e longo prazo. Cada uma destas características lean serão ilustradas em gráficos simulados da LOB. O trabalho conclui afirmando que Linha de Balanço é Lean, propiciando discussões acerca desta síntese conceitual.
\end{abstract}

Palavras-chave: Linha de balanço, programação de obra, lean construction, síntese dos conceitos lean, comunicação gráfica dos conceitos lean.

\begin{abstract}
Evidence on the use of Line of Balance as a scheduling technique already exists in the Brazilian construction industry since thirty years ago. More recently it has been associated with Lean Construction applications, especially as tool for strategic planning of works. This conceptual paper discusses how line of balance can be taken not just as a straightforward graphical device to depict site programming, but in such a way that philosophical concepts behind lean production there may be incorporated or make selfevident. Some characteristics of lean production can be applied to the development of the LOB, like cycle in time and space, streamlining operations by outsourcing and work packaging, variability reduction, flow, lead time reduction by balancing of the activities and learning effect and integration of short, medium and long term. Each of these lean features will be illustrated on simulated LOB graphs. The paper concludes that Line of Balance is Lean, providing discussions of this conceptual synthesis.
\end{abstract}

Keywords: Line of balance, building schedules, lean construction, lean conceptual synthesis, graphical communication of lean principles.

\section{INTRODUÇÃO}

Métodos de planejamento e controle baseados em Redes, como o Método do Caminho Crítico (CPM), são utilizados desde a década de 1950 como ferramenta de planejamento e controle da produção de obra, porém não são adequados para obras de natureza repetitiva, nas quais métodos alternativos de planejamento como programação linear e 
baseada na localização são mais eficazes (ARDITI; TOKDEMIR; SUH, 2002; LUCKO; ALVES; ANGELIM, 2013).

Estes métodos lineares são estudados desde a década de 1970 (ARDITI; ALBULAK, 1986) sendo chamados genericamente de "Métodos de Planejamento Linear". Entretanto há indícios de uso de tais técnicas na década de 1950 (PACHECO; HEINECK, 2008). A técnica de planejamento Linha de Balanço (Line of Balance LOB) é utilizada como ferramenta de programação de obras repetitivas, principalmente de edifícios (MENDES JR.; HEINECK, 1999; TOMMELEIN; BALLARD, 1997).

Um novo paradigma de produção surgiu em contraste ao conhecido e bem disseminado paradigma da produção em massa desenvolvido no período do taylorismo e fordismo (WOMACK ET AL., 1992; FORMOSO, 2002). Este paradigma foi nomeado de Lean Production, Produção Enxuta, e objetiva a eliminação de desperdícios na produção. Koskela (1992) avaliou a aplicação da produção enxuta na construção civil, quando o denominou Lean Construction traduzido como Construção Enxuta que é uma proposta de nova filosofia de gestão de produção aplicada na construção civil.

Trabalhos assinalam o uso da LOB apenas como uma ferramenta de planejamento na implantação da construção enxuta (SOUZA ET AL., 2005; BARBOSA ET AL., 2013), mas há autores que exploraram a Linha de Balanço como uma ferramenta que detém várias características lean (MATOS ET Al., 2009; MONTEIRO ET Al., 2011; CARNEIRO ET AL., 2012; LUCKO ET AL., 2013; MOURA; MONTEIRO; HEINECK, 2014).

Com isso, a questão da pesquisa é se os conceitos e características da construção enxuta podem ser incorporados no planejamento por LOB. De forma específica se estes são facilmente visualizados nos gráficos da LOB. Assim o objetivo do trabalho é identificar quais destes conceitos podem ser inseridos na etapa de planejamento para obras repetitivas por LOB, tornando-a uma ferramenta de uso preferencial com essa nova filosofia de produção.

\section{FUNDAMENTAÇÃO TEÓRICA}

\subsection{Construção Enxuta}

A produção enxuta foi originalmente proposta em 1990 por Womack e Jones (WOMACK; JONES, 1992). O conceito de construção enxuta surgiu em 1992 com o trabalho seminal de Koskela, quando nasceram seus onze princípios para aplicação da produção enxuta na construção. Womack e Jones (1996) apresentaram a evolução dos conceitos desta produção nos cinco princípios da Mentalidade Enxuta. Heineck et al. (2009) os reduziram a três: ciclo, fluxo e coordenação. O primeiro conduz a uma redução do tamanho do lote mediante a transformação das atividades a executar em ciclos repetitivos, o segundo se reflete em operações que não param e tenham uma sequência mais contínua possível e o terceiro foca na viabilização dos conceitos lean pela coordenação das atividades.

Nota-se um consenso entre os vários autores de que a filosofia da construção enxuta tem como principal foco criar valor para os clientes, melhorar as operações em pequenos passos e continuamente com o objetivo de reduzir os desperdícios, seja de tempo, material ou dinheiro. 


\subsection{Linha de balanço}

No Brasil encontra-se na literatura referência do uso desde em 1981 (MACEDO, 1981), sendo somente na década de 1990 seu uso mais frequente (HEINECK; PEIXE, 1990; LOSSO; ARAUJO, 1995; HEINECK, 1996).

Esta ferramenta considera as sequências das atividades pelas diversas repetições das unidades da obra (pavimento, apartamento, casas unifamiliares, quilômetros de estrada ou metros de canalização, por exemplo) em relação a unidades de tempo, preservando constante a taxa de saída ou produção por unidade de tempo (SARRAJ, 1990). A partir da adoção desse conceito, as atividades seguem ritmos de produção definidos, em que todas as atividades da obra são realizadas em um só ritmo. A busca da eliminação do tempo ocioso entre as atividades, sugerindo que todas tenham ritmos parecidos (balanceados) é o objetivo da LOB (MENDES JR.; HEINECK, 1999).

O balanceamento adequado das atividades na LOB assegura a continuidade dos serviços, sincronismo, apropriado tempo de folga, redução da variabilidade com melhor utilização dos recursos e mão de obra, maior garantia do prazo de finalização da obra, além de aumento da transparência do processo (DEPEXE ET AL., 2006).

A transparência alcançada pela visualização da linha de balanço pode ser prejudicada pela quantidade de linhas no gráfico, pois podem ser muitas em planejamento de grandes obras com muitas atividades. Esta desvantagem é contornada pela utilização de gráficos coloridos (ARDITI; ALBULAK, 1986) ou formação de células de produção. Uma revisão atualizada de artigos de LOB foi realizada por Lucko et al. (2013) em um trabalho que objetiva preencher a lacuna de conhecimento sobre estudos quantitativos de produtividade em planejamento por linha de balanço.

\section{METODOLOGIA}

$\mathrm{O}$ artigo foi desenvolvido através de uma revisão da literatura dos conceitos referentes à construção enxuta e à técnica de programação de linha de balanço.

A pesquisa bibliográfica foi realizada principalmente em artigos nacionais do SIBRAGEC (Simpósio Brasileiro de Gestão e Economia da Construção) e ENTAC (Encontro Nacional de Tecnologia no Ambiente Construído), além de eventos e periódicos internacionais. Resultou na criação de gráficos que visam traduzir em forma de imagem os conceitos lean integrados na linha de balanço.

\section{CONSTRUÇÃO ENXUTA NA PROGRAMAÇÃO POR LOB}

Algumas características da construção enxuta podem ser aplicadas no desenvolvimento da LOB, tais como a visão do ciclo no tempo e no espaço, simplificação das operações pela externalização ou pela pacotização do trabalho, redução da variabilidade, visão do fluxo de execução, redução do lead time pelo balanceamento das atividades ou pelo efeito aprendizagem, assim como integração de curto, médio e longo prazo. Cada uma destas características lean serão ilustradas em gráficos (Figuras 1 a 8) simulados da LOB.

\subsection{Ciclo no tempo e espaço}

Ciclo pode ser considerado como a repetição de atividades no tempo, no espaço ou conjuntamente no tempo e espaço, conforme ilustrado na Figura 1 as divisões na 
horizontal e na vertical determinam um ciclo. Para a adoção de práticas lean, as atividades devem ser executadas em ciclos com tamanhos compatíveis para poderem ser repetitivos (HEINECK ET AL., 2009).

A partir do conceito de ciclo é possível simplificar atividades, diminuir as interfaces, juntas e ligações, reduzir a variabilidade, reduzir desperdício, reduzir as atividades que não agregam valor ao processo, comprimir o tempo de ciclo e realizar lotes pequenos que tendem ao lote unitário e ajudam a preparar os trabalhos no canteiro. Na linha de balanço é possível fazer a explosão das atividades e insumos (exemplificado pela simplificação das atividades), facilitando o planejamento de curto e médio prazo conforme será ilustrado nas figuras seguintes.

Figura 1 - Formas de apresentação dos ciclos no tempo e espaço

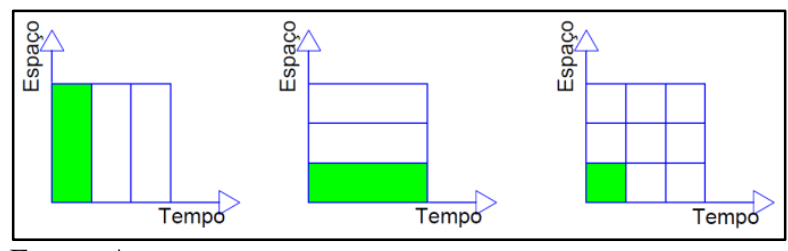

Fonte: Autores

\subsubsection{Simplificação das operações}

Simplificação das operações é a redução do número de componentes de um produto, de número de partes ou estágios num fluxo de materiais ou informações, ou seja, é obtida através da diminuição de interfaces, juntas e ligações entre os processos (ISATTO ET AL., 2000; BERNARDES, 2003). Quanto maior o número de componentes ou passos em um processo, maior tende a ser o número de atividades que não agregam valor (ISATTO ET AL., 2000), com isso a simplificação ajuda na eliminação destas atividades.

Simplificação pode ser alcançada através da pacotização do trabalho (formação de célula de produção) que reduz o tempo de set-up (Figura 2) e externalização do processo produtivo, permitindo maior participação de fornecedores em relação ao processo produtivo, como montagem de estruturas fora do canteiro de obra e entregues no momento de utilização indicado pela LOB (Figura 3). A pacotização do trabalho permite uma sequência flexível de montagem e execução das atividades e pela aglutinação destas, a célula de produção passa a ser o caminho crítico, fazendo com que os trabalhadores se auto ajustem na execução das atividades.

Figura 2 - Pacotização do trabalho (célula de produção)

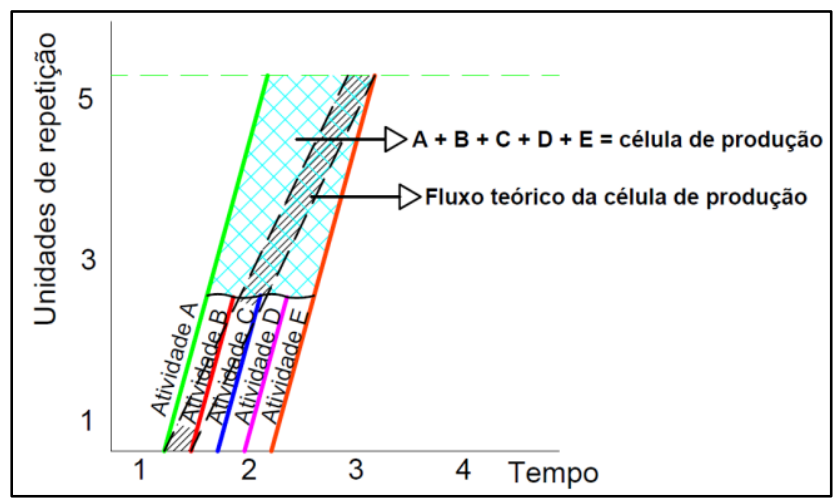

Fonte: Autores 
A externalização do processo de produção acontece quando partes do trabalho são retiradas do canteiro de obra para serem executadas externamente e entregues na obra no momento de montagem.

Figura 3 - Externalização do processo de produção

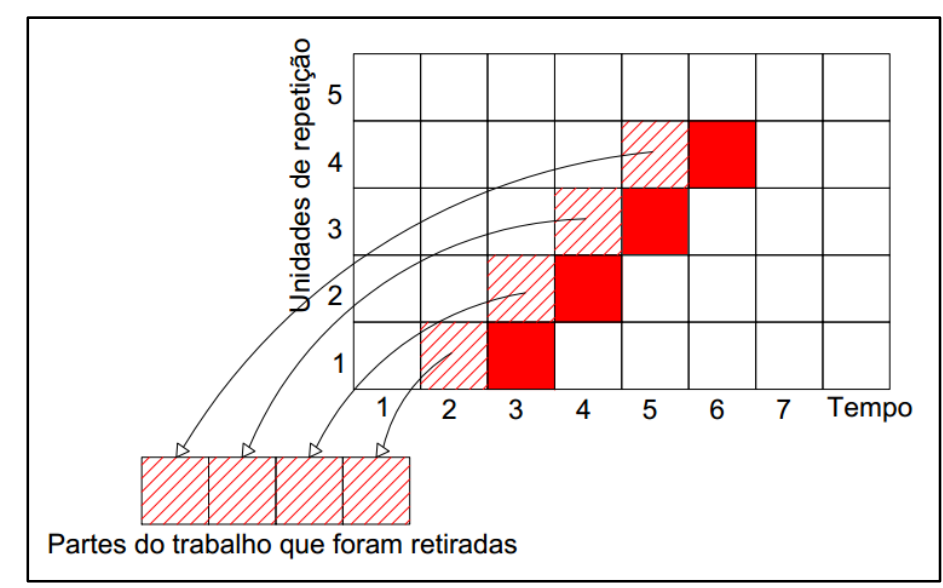

Fonte: Autores

\subsubsection{Redução da variabilidade}

Depexe et al. (2006) relatam que com o uso da linha de balanço é possível reduzir a variabilidade no processo de produção de edifícios. Existem três tipos de variabilidade: variabilidade nos processos anteriores, variabilidade no próprio processo e variabilidade na demanda (ISATTO ET AL., 2000). A primeira está relacionada com os fornecedores do processo, a segunda à execução do processo e a terceira aos desejos dos clientes do processo. A variabilidade na execução de uma determinada atividade pode ser um exemplo da segunda variabilidade e está representada na Figura 4 que é resolvida com a padronização dos ciclos na programação por LOB.

Figura 4 - Variabilidade no processo e padronização dos ciclos

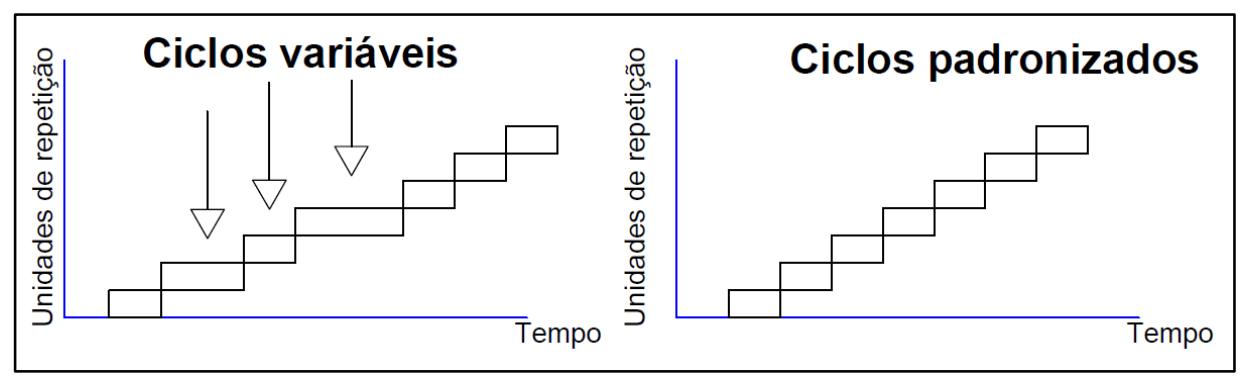

Fonte: Autores

\subsection{Visão do fluxo de execução}

Fluxo pode ser definido como o movimento contínuo, caracterizado pelo ritmo de ataque às unidades de repetição que na construção enxuta é utilizado como takt time da atividade. O modelo de produção enxuta é definido como fluxo de materiais e/ou informações que vão desde a matéria prima até o produto final (KOSKELA, 1992). O fluxo contínuo das atividades pode ser mantido pela eliminação das restrições e das atividades que não agregam valor. A vantagem da visualização do fluxo na LOB é a transparência no processo, facilitando a detecção de erros, diminuindo a necessidade de inspeção dos produtos ao final da linha de produção (PINHEIRO, 2009), além de 
detectar a superposição das atividades. A Figura 5 mostra uma simulação de planejamento por LOB enfatizando a fácil visualização do fluxo das atividades e o takt time, dando mais visibilidade do trabalho.

\section{Figura 5 - Visualização do fluxo de execução de atividades na LOB}

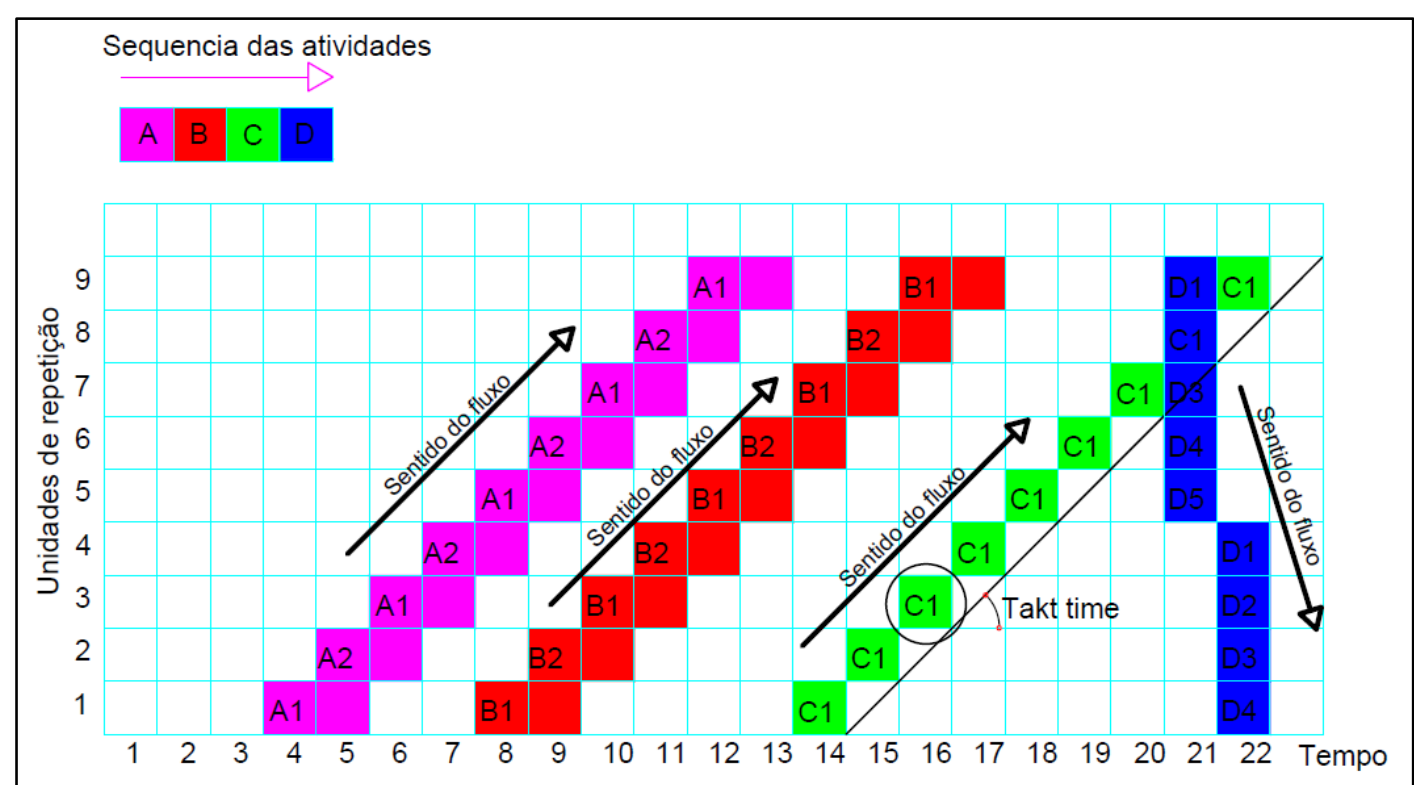

Fonte: Autores

\subsection{Redução do lead time}

Lead time é o tempo necessário para que todas as atividades ocorram em uma unidade de repetição (PACHECO; HEINECK, 2008). Porém pode ser o tempo de operação de cada atividade em uma unidade de repetição, lead time da operação, ou o tempo total de operação de todas as atividades em todas as unidades de repetição. Conjugando a unidade de repetição com o efeito aprendizagem, o tempo de ciclo da atividade na programação por LOB é diminuído. Assim a escolha de unidades de repetição de menor dimensão amplifica o efeito aprendizagem permitindo a redução do tempo de ciclo (PACHECO; HEINECK, 2008). Uma vez que os efeitos da aprendizagem sejam ligeiramente diferentes, as curvaturas de aprendizagem serão diferentes como mostra a Figura 6.

Figura 6 - Redução do lead time pelo efeito aprendizagem

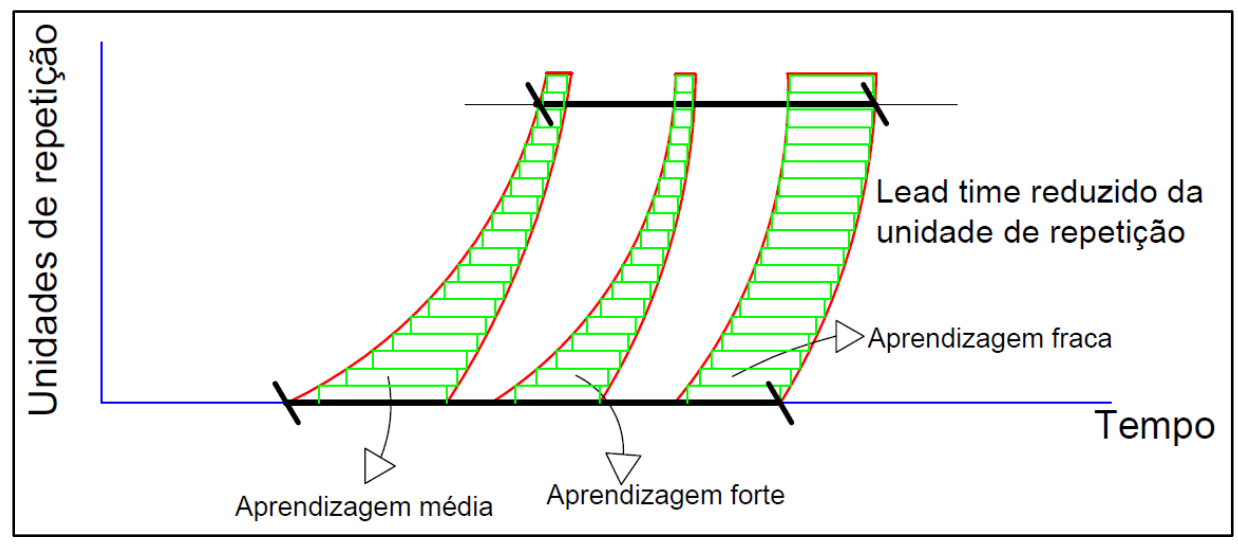

Fonte: Autores 
O lead time pode também ser reduzido pelo ajuste dos ritmos das atividades e redução dos tempos de folga (buffers), alcançado pela adequação e balanceamento da equipe de trabalho.

Figura 7 - Redução do lead time pelo balanceamento das atividades

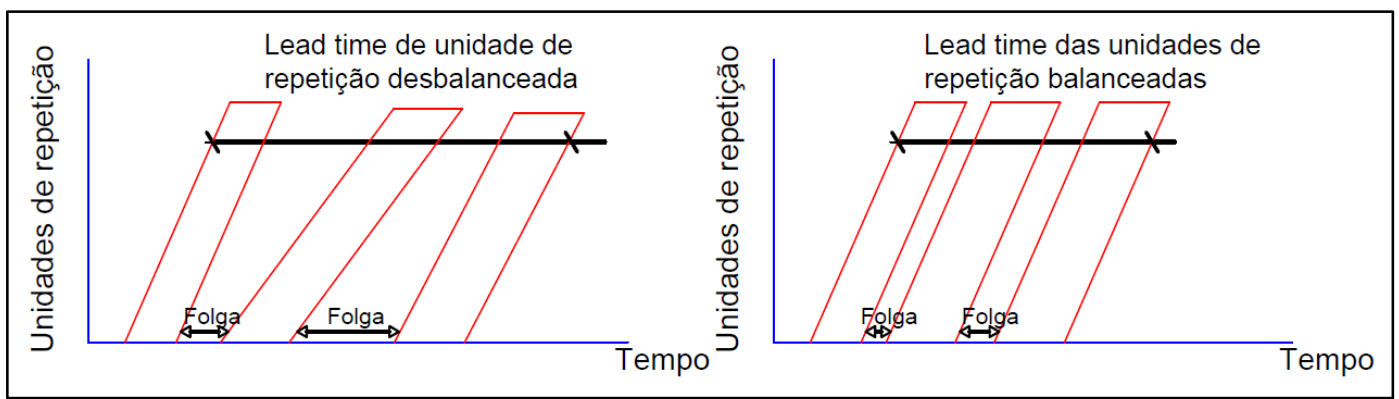

Fonte: Autores

\subsection{Integração de curto, médio e longo prazo.}

A técnica da linha de balanço é uma ferramenta usada para planejamento de longo prazo, mas é possível integrar as visões de médio e curto prazo das atividades a serem realizadas como mostra a Figura 8. Atividades de preparação podem ser inseridas na programação por linha de balanço, tais como melhoria da capacidade de produção através de fornecimento de materiais e ferramentas ou treinamento, atividades auxiliares como viabilização de transporte, que fazem parte do planejamento de médio prazo, onde são removidas as restrições. A programação de curto prazo é derivada da programação de médio prazo e pode incluir antecipações de menor porte e também atividades de setup que possam fazer parte do ciclo das operações.

\section{Figura 8 - Integração de curto, médio e longo prazo}

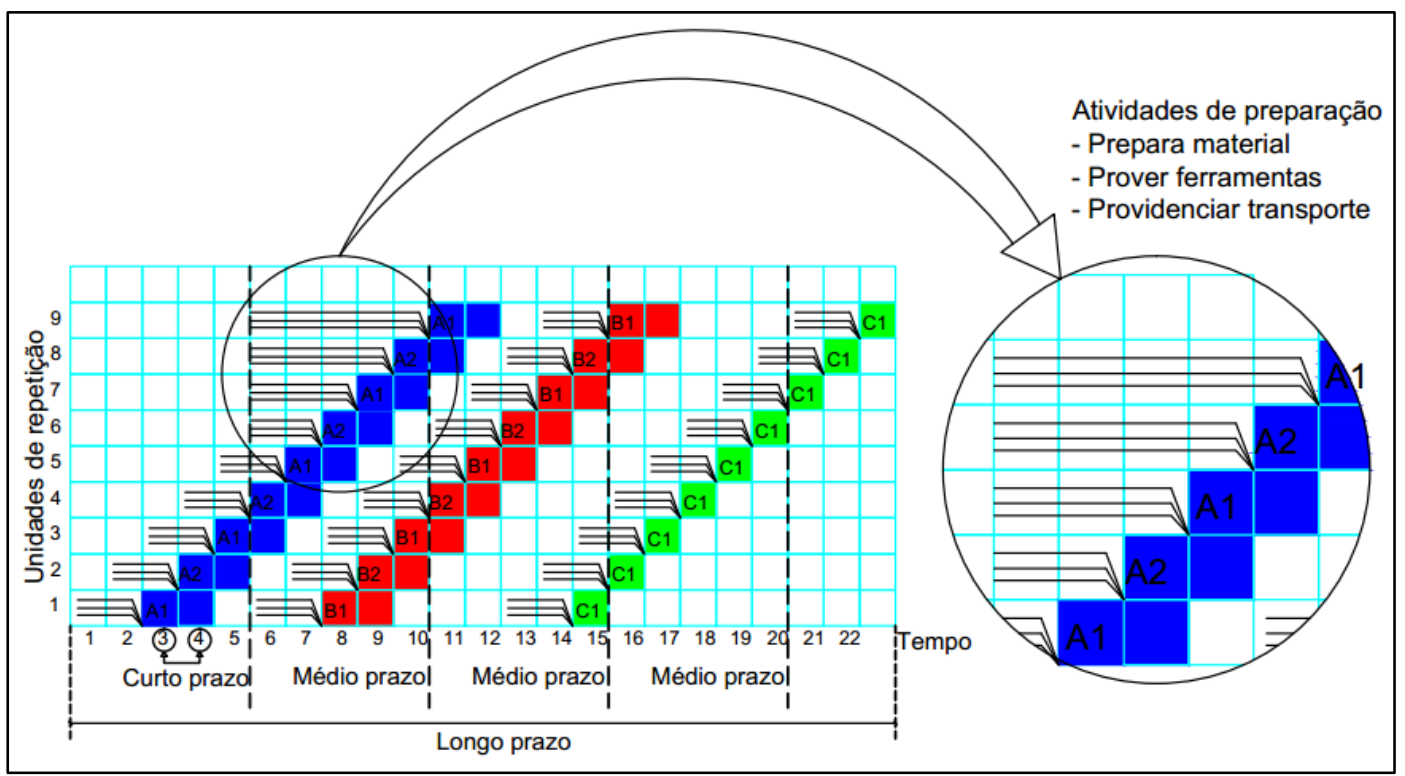

Fonte: Autores 


\section{CONCLUSÕES}

O artigo revisa vários conceitos da filosofia Lean, indicando a importância de atividades em ciclo e que facilitam o fluxo de informação e entrega das unidades acabadas da construção. Algumas considerações para o planejamento da linha de balanço Lean são estudadas, visão de ciclo no tempo e espaço, simplificações das operações, redução da variabilidade, visão do fluxo de execução, redução do lead time e integração do curto, médio e longo prazo.

A pesquisa corrobora com a influência construção enxuta na otimização da produção na construção, reconhecendo a LOB como uma ferramenta de planejamento que confirma a filosofia Lean, carregando consigo conceitos que visam maximizar o fluxo contínuo, mantendo o ciclo das operações de maneira coordenada.

Por fim, foram ilustrados os conceitos em gráficos na forma de LOB que fazem parte da filosofia da construção enxuta e devem ser tomados como base para planejamento lean. Conclui-se que linha de balanço é lean. Espera-se que mais conceitos lean visualizados na LOB sejam gerados pela comunidade acadêmica, de maneira que possam ser discutidos e aperfeiçoados.

\section{AGRADECIMENTOS}

Os autores agradecem à Coordenação de Aperfeiçoamento de Pessoal de Nível Superior - CAPES pelo suporte financeiro e à Universidade Federal do Ceará e Universidade Estadual do Ceará pelo suporte institucional necessário para o desenvolvimento deste artigo.

\section{REFERÊNCIAS}

ARDITI, D.; ALBULAK, Z. Line-of-balance scheduling in pavement construction. Journal of Construction Engineering and Management, 1986, 112: 411-424.

ARDITI, D.; TOKDEMIR, O. B.; SUH, K. Challenges in line-of-balance scheduling, Journal of Construction Engineering and Management, 2002, 128: 545-556.

BARBOSA, G.; ANDRADE, F.; BIOTTO, C.; MOTA, G. Implementação de construção enxuta em um ano em um projeto de construção. In: SIMPÓSIO BRASILEIRO DE GESTÃO E ECONOMIA DA CONSTRUÇÃO, 8., 2013, Salvador. Anais... Salvador, 2013.

BERNARDES, M. M. S. Planejamento e controle da produção para empresas de construção civil. Rio de Janeiro: LTC Editora, 2003.

CARNEIRO, T. M.; GONÇALVES, P. P.; HEINECK, L. F. M. Análise de como puxar a produção em canteiros repetitivos planejados com linha de balanço. In: ENCONTRO NACIONAL DE TECNOLOGIA DO AMBIENTE CONSTRUIIDO, 14., 2012, Juiz de Fora. Anais... Juiz de Fora, 2012.

DEPEXE, M. D.; MELO, M. C. de; DORNELES, J. B.; KEMMER, S. L.; HEINECK, L. F. M. Aplicação das práticas da Linha de Balanço segundo os princípios da Lean Construction. In: ENCONTRO NACIONAL DE TECNOLOGIA DO AMBIENTE CONSTRUÍDO, 11., 2006, Florianópolis. Anais... Florianópolis, 2006. 
FORMOSO, C. T. Lean Construction: princípios básicos e exemplos. Pini, 10 out. 2002. Disponível em: <www.piniweb.com.br/construcao/noticias/lean-constructionprincipios-basicos-e-exemplos-80714-1.asp>. Acesso em: 29 nov. 2013

HEINECK, L. F. M.; ROCHA, F. E. M. da; PEREIRA, P. E.; LEITE, M. O.

Introdução aos Conceitos Lean: Visão Geral do Assunto. V. 1. Fortaleza: Expressão Gráfica Editora. 2009.

HEINECK, L. F. M.; PEIXE, L. T. Aplicação do conceito do método de linha de balanço à programação de obras repetitivas: decisões fundamentais para sua aplicação. In: ENCONTRO NACIONAL DE ENGENHARIA DE PRODUÇÃO, 10., 1990, Belo Horizonte. Anais... Belo Horizonte, 1990.

ISATTO, E. L.; FORMOSO, C. T.; CESARE, C. M. de; HIROTA, E. H.; ALVES, T. da C. L. Lean Construction: diretrizes e ferramentas para o controle de perdas na Construção Civil. Porto Alegre: SEBRAE/RS, 2000.

KOSKELA, L. Application of the New Production Philosophy to Construction. Stanford University, CIFE Technical Report \# 72, 1992. 87 p.

LOSSO, I. R.; ARAÚJO, H. N. Aplicação do método da linha de balanço: estudo de caso. In: ENCONTRO NACIONAL DE TECNOLOGIA DO AMBIENTE CONSTRUÍDO, 1995. Anais... Rio de Janeiro, 1995.

LUCKO, G.; ALVES, T. da C. L.; ANGELIM, V. L. Challenges and opportunities for productivity improvement studies in linear, repetitive, and location-based scheduling. Construction Management and Economics, 2013. DOI: 10.1080/01446193.2013.845305.

MACEDO, M. L. de. A aplicação do método da linha de balanço na coordenação da execução de canteiros de habitações unifamiliares. In: Simpósio Latino-Americano de Racionalização da Construção e sua Aplicação às Habitações de Interesse Social, 1981, São Paulo. Anais... São Paulo, 1981, p. 777-786.

MATOS, A. O.; FERREIRA, E. de A. M.; SANTOS, D. G. Princípios da construção enxuta aplicada à programação de uma obra por linha de balanço. In: SIMPÓSIO BRASILEIRO DE GESTÃO E ECONOMIA DA CONSTRUÇÃO, 7., 2009, João Pessoa. Anais... João Pessoa, 2009.

MENDES JR., R.; HEINECK, L. F. M. Towards production control on multi-story building construction sites. In: ANNUAL CONFERENCE OF THE INTERNATIONAL GROUP FOR LEAN CONSTRUCTION, 7., 1999, Berkeley. Proceedings... Berkeley, 1999, p. 313 - 324.

MONTEIRO, J. M. F.; CRUZ, A. C. de M.; MOREIRA, K. M. de V.; CRUZ, L. T. G.; MORORÓ, M. S. de M.; HEINECK; L. F. M. Identificação gráfica de elementos da construção enxuta no planejamento de obras com Linha De Balanço. In: SIMPÓSIO BRASILEIRO DE GESTÃO DA QUALIDADE E ORGANIZAÇÃO TRABALHO NO AMBIENTE CONSTRUÍDO, 7., 2011, Belém. Anais... Belém, 2011.

MOURA, R. de S. L. M.; MONTEIRO, J. M. F.; HEINECK, L. F. M. Line of Balance Is it a synthesis of Lean Production principles as applied to site programming of works? 
In: ANNUAL CONFERENCE OF THE INTERNATIONAL GROUP FOR LEAN CONSTRUCTION, 22., 2014, Oslo. Proceedings... Oslo, 2014, p. $703-714$.

PACHECO, M. T. G.; HEINECK, L. F. M. Redução do tempo de atravessamento em programação por linha de balanço através da redução da unidade de repetição sobre influência do efeito aprendizado: uma visão enxuta. In: ENCONTRO NACIONAL DE TECNOLOGIA DO AMBIENTE CONSTRUIIDO, 12., 2008, Fortaleza. Anais... Fortaleza, 2008.

PINHEIRO, M. B. Considerações gráficas sobre a ligação entre a Linha de Balanço e o Sistema Toyota de Produção. Monografia. Universidade Federal do Ceará, Fortaleza, CE, 2009.

SARRAJ, Z. M. A. Formal Development of Line-of-Balance Technique. Journal of Construction Engineering and Management, 1990, 116: 689-704.

SOUZA, D. P de; BASTOS, M. R.; BARROS NETO, J. de P.; MOURA, S. M. de; PEREIRA, P. E.; HEINECK, L. F. M. Uma metodologia e implantação do Sistema Toyota de Produção em uma empresa de construção de edifícios a partir do suporte tecnológico do Programa de Inovação da Construção Civil do Ceará (INOVACON-CE). In: SIMPÓSIO BRASILEIRO DE GESTÃO E ECONOMIA DA CONSTRUÇÃO, 4. 2005, Porto Alegre. Anais... Porto Alegre, 2005.

TOMMELEIN, I. D.; BALLARD, G. Look ahead planning: screening and pulling. In: SEMINÁRIO INTERNACIONAL SOBRE LEAN CONSTRUCTION, 2., 1997, São Paulo. Anais... Instituto de Engenharia de São Paulo/Logical Systems, 1997.

WOMACK, J. P.; JONES D. T. A Mentalidade Enxuta nas empresas: elimine o desperdício e crie riqueza. 5. ed. Rio de Janeiro: Campus, 1996.

WOMACK, J. P.; JONES, D. T.; ROOS, D. A máquina que mudou o mundo. Rio de Janeiro: Campus, 1992. 